\title{
OP-26 KNOWLEDGE LEVELS OF RSBY BENEFICIARIES IN JHAGADIA, GUJARAT
}

Deepika Saluja. IIMA - Indian Institute of Management Ahmedabad, Ahmedabad (Gujarat), India

\subsection{6/bmjgh-2016-EPHPabstracts.26}

Background Approximately $90 \%$ of the Indian population is working in the unorganised sector. Major concerns for this population are absence of health coverage and financial insecurity in case of medical expenditure. The 2007 National Sample Survey noted that household earnings, savings and borrowings collectively contribute around $94 \%$ to total medical expenditures. In 2008, the government of India launched Rashtriya Swasthya Bima Yojana (RSBY), providing health insurance coverage to the weaker sections of society with the objective of improving access to quality health services while reducing financial burden at household level. The present study aims at understanding the knowledge levels of RSBY beneficiaries about the scheme and the issues they are facing in accessing the scheme.

Methods Research participants were 60 RSBY beneficiaries and their accompanying family members who came for treatment to SEWA Rural hospital, Jhagadia (Gujarat), in May and June 2012. Informed consent was obtained, semi-structured interviews were conducted with the participants and field notes were taken. The data were analysed in a mixed-methods approach, based on qualitative coding supported by descriptive statistics.

Findings A majority of the participants labelled the scheme in ways like "the card which can be used for getting treatment for illnesses", "the card in which photos and fingerprints are taken" or "dawakhana [hospital] card".

Very few respondents knew the basics of the scheme, not even the name RSBY. When asked for knowledge on the scheme, only one in four of the patients were able respond. In that case their family members, primarily spouses, responded. The respondents' age ranged between 18 and 80 and education levels ranged from completely illiterate to 12 th grade. Of the patients three-fourth were females, of the respondents three-fourth were males. Respondents were largely unaware of the scheme details such as the amount of insurance coverage (43\%), the disease covered (48\%), the maximum number of household members covered (57\%), the amount left in the card (76\%), the hospitals empanelled (92\%), and the possibility of card details modification $(95 \%)$. On the other hand, awareness was high on yearly card renewal (82\%), on the enrolment fee charged $(90 \%)$, and on the name of the main beneficiary in the card (95\%).

The issues beneficiaries are facing when accessing RSBY could be categorized as related to prior to enrolment, enrolment, and post-enrolment. Prior to enrolment, the important issues revealed were poor IEC (Information, Education and Communication) activities and conflicting interests of insurance companies, combined with low literacy levels of the potential beneficiaries. As pre-enrolment, enrolment and post-enrolment are consecutive steps, it is prior to enrolment where improved 
knowledge levels can enhance RSBY's uptake. All further steps become dependent on the effectiveness of IEC. During the enrolment phase, apart from low enrolment due to low awareness, there are issues with fingerprint mismatch, wrong information entered in the card, and misprinted cards. Post-enrolment, issues faced are often again related to poor awareness levels (like no knowledge on the district kiosk or on the toll-free number to get grievances resolved). Such can be prevented by providing timely information during pre-enrolment and enrolment stages, and reconfirms the need for effective IEC activities to make the scheme implementation successful.

The issues faced can alternatively be categorized into technical and design issues. Technical issues include non-readability of card, thumb impression mismatch, and wrong beneficiary details. Design issues include illnesses not covered, ineffective grievance redressal, insufficient checks and balances, and above all poor IEC activities.

Discussion \& recommendations Given the importance of RSBY for the targeted population and four years into the implementation; understanding of the nature of the issues faced by RSBY beneficiaries is key for finding plausible solutions. Though knowledge levels vary drastically among the indicators, awareness and knowledge on essential details of the scheme are low. Interestingly, we found that most of the communication (good or bad) on RSBY within the community happened through word of mouth route via Anganwadi workers, ASHA's and neighbours. By contrast, IEC activities as currently developed were largely ineffective.

Respondents explicitly demanded more and more relevant informant to be able to use the scheme to its maximum. We recommend however information not be loaded all at once during enrolment. Rather, it needs to be provided in a systematic manner at different junctures of the beneficiaries' interactions with the policy. The information must be reinforced using multiple channels to keep reminding the beneficiaries about scheme benefits.

Ineffective IEC activities being related to conflict of interest of the insurers, we recommend accountability mechanisms to be incorporated in the system. Clearly defining the protocol to conduct IEC activities with measurable parameters (currently absent in the policy) and linking them to the premium to give it financial reinforcement appears promising in the present context. Reducing ambiguity in the contract and dependency on a few actors, and strengthening accountability in the system is the way forward to bring RSBY's policy objectives closer.

No competing interest. 